\title{
RADIOGRAPHIC ABNORMALITIES OF THE TALUS IN PATIENTS WITH CLUBFOOT AFTER SURGICAL RELEASE USING THE MCKAY TECHNIQUE
}

\author{
José Antonio Pinto', Andréa Canizares Hernandes², Thais Paula Buchaim², Francesco Camara Blumetti ${ }^{3}$, Carla Chertman $^{4}$, \\ Patrícia Corey Yamane ${ }^{5}$, Artur da Rocha Corrêa Fernandes ${ }^{6}$
}

\begin{abstract}
Objective: To analyze morphological abnormalities of the talus in patients with clubfoot after surgical treatment using the McKay technique. Method: Lateral standing-position radiographs of the feet of 14 patients with unilateral clubfoot who underwent treatment by means of the doubleincision McKay technique were retrospectively analyzed. All the patients were operated by the same surgeon, with an average of 6.53 years between surgery and the radiograph. We compared the radiographic characteristics of the talus between the operated and the contralateral foot. We assessed the presence of deformity of the talar dome and head (sphericity evaluation); the talar length and height; the percentage and degree of navicular subluxation; abnormalities of the Gissane angle; and the trabecular bone pattern. Results: Abnormalities of the talar head occurred
\end{abstract}

in $92.8 \%$ of the patients; of the talar dome in $92.8 \%$; and of the trabecular pattern in $100 \%$. The talar length ratio between the operated and the contralateral foot ranged from 0.61 to 0.88 (mean $0.79 ; \mathrm{SD}=0.09$ ), while the height ratio ranged from 0.57 to 0.98 (mean $0.82 ; \mathrm{SD}=0.12$ ). The Gissane angle was greater in all of the operated feet, and all of them also showed navicular subluxation, at a rate ranging from 6.43 to $59.75 \%$ (mean $26.34 \%$; $\mathrm{SD}=16.66 \%$ ). Conclusion: Talar abnormalities occurred in $100 \%$ of the feet treated using the McKay technique. It was shown that establishing radiographic parameters to describe and quantify these deformities was feasible, through simple and easy-to-perform techniques.

Keywords - Clubfoot/radiography; Talus; Surgical Procedures, Operative; Morphology

\section{INTRODUCTION}

Equinocavovarus clubfoot is characterized by a deformity caused mainly by misalignment and malformation of the bones and joints of the hindfoot. Its mean incidence in the population is $1: 1000$ live births $^{(1)}$ and it is one of the congenital abnormalities most studied by the orthopedic scientific community.

In a search conducted in the Brazilian and worldwide literature, we found around 700 articles on this topic published over the last ten years. However, the specific coverage of complications relating to radiographic deformities found in the talus during and after treatment was significantly smaller in quantity, comprising simple citations or paragraphs within studies that had other focuses.

It is known that talus deformities generally result in varying degrees of necrosis, a complication that usually goes unnoticed during treatment for clubfoot. Its incidence is greater when the treatment is surgical,

1 - Adjunct Professor and Head of the Discipline of Pediatric Orthopedics, Department of Orthopedics and Traumatology, Federal University of São Paulo, SP, Brazil.

2 - Resident Physician, Department of Orthopedics and Traumatology, Federal University of São Paulo, SP, Brazil.

3 - Attending Physician, Discipline of Pediatric Orthopedics, Department of Orthopedics and Traumatology, Federal University of São Paulo, SP, Brazil.

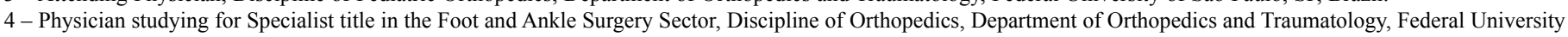
of São Paulo, SP, Brazil.

5 - Attending Physician, Discipline of Pediatric Orthopedics, Department of Orthopedics and Traumatology, Federal University of São Paulo, SP, Brazil.

6 - Adjunct Professor and Deputy Head of the Department of Imaging Diagnostics, Federal University of São Paulo, SP, Brazil.

Work performed in the Discipline of Pediatric Orthopedics, Department of Orthopedics and Traumatology, São Paulo Medical School, Federal University of São Paulo. correspondence: Andréa Canizares Hernandes, Rua Borges Lagoa, 783, $5^{\circ}$ andar - Vila Clementino - 04038-032 - São Paulo, SP.

E-mail: andrea_epm70@ig.com.br

Work received for publication: June 2, 2010; accepted for publication: November 29, 2010. 
especially when the technique used requires large release of soft tissue. However, descriptions of the "flat top" talus deformities that are classically correlated with necrosis can also be found in patients undergoing nonsurgical treatment. This deformity is probably caused by forced dorsiflexion of the foot, in an attempt to reduce the clubfoot condition, thereby generating a lever mechanism that deforms the flexible cartilaginous mold of this bone ${ }^{(2)}$.

The talus is vascularized solely through vessels penetrating the bone by means of capsular and ligamentous insertions, which makes it particularly susceptible to avascular necrosis. Its arterial supply from outside of the bone basically comes from branches of the posterior tibial, anterior tibial and fibular arteries $^{(3)}$. These vessels form several anastomotic plexi around the talus. The calcaneal branch of the posterior tibial artery forms a network with the fibular branch, which irrigates the posterior tuberosity of the talus. The biggest artery of the tarsal canal, also coming from the posterior tibial artery, forms a rich vascular plexus with the tarsal sinus artery (which presents contributions from the anterior tibial artery and fibular artery). These anastomoses occur inside the tarsal canal and irrigate most of the central body and lateral region of the talus. A smaller deltoid branch arises at the origin of the tarsal canal artery and runs between the talotibial and talocalcaneal parts of the deltoid ligament to supply the medial part of the talus body. The anterior tibial artery issues branches to the head and neck of the talus, with contributions from the fibular artery and the deltoid branch of the posterior tibial artery.

From knowledge of the circulatory supply, it can be concluded that the techniques that provoke greatest injury are the ones that advocate peritalar release, especially with sectioning of the deltoid ligament. The McKay and Simons technique consists of complete subtalar release, with optional sectioning of the talocalcaneal interosseous ligament, stretching of the flexor tendons, complete sectioning of the superficial deltoid ligament, partial sectioning of the deep deltoid ligament and sectioning of the bifurcated, plantar calcaneonavicular and dorsal talonavicular ligaments. This procedure can be carried out by making a classical Cincinnati incision or a double (lateral and medial) incision ${ }^{(4)}$. Under these circumstances, precise anatomical knowledge is necessary, so that the chances of osteonecrosis are minimized.

\section{OBJECTIVE}

The aim of our study was to analyze the morphological alterations of the talus resulting from surgical treatment for clubfoot by means of peritalar release.

\section{MATERIAL AND METHOD}

The medical records of 76 patients with clubfoot attended at a public hospital in São Paulo were analyzed retrospectively. In order to standardize our sample, we only included patients with unilateral clubfoot who underwent surgical treatment by means of the McKay technique, with double incision, between 1996 and 2008. All the procedures were performed by the same orthopedist. A total of 14 patients met the inclusion criteria and were evaluated in this study. Their general characteristics are summarized in Table 1.

Table 1 - Data on patients, including age, sex, side affected, date of surgery and date of the radiograph evaluated.

\begin{tabular}{c|c|c|c|c|c}
\hline Patient & Age & Sex & Side & $\begin{array}{c}\text { Date of } \\
\text { surgery }\end{array}$ & $\begin{array}{c}\text { Date of } \\
\text { radiograph }\end{array}$ \\
\hline 1 & 11 & M & L & 1990 & Dec 12, 2006 \\
\hline 2 & 7 & M & L & 2003 & Nov 28, 2007 \\
\hline 3 & 13 & M & R & 1996 & Apr 10, 2008 \\
\hline 4 & 8 & M & L & 2002 & Mar 5, 2008 \\
\hline 5 & 7 & M & L & 2002 & Nov 9, 2005 \\
\hline 6 & 7 & F & L & 2003 & Nov 4, 2005 \\
\hline 7 & 10 & M & L & 2001 & Feb 7, 2007 \\
\hline 8 & 11 & M & L & 2002 & Jun 6, 2008 \\
\hline 9 & 11 & M & R & 1999 & Feb 12, 2009 \\
\hline 10 & 14 & M & L & 1999 & Mar 10, 2006 \\
\hline 11 & 8 & F & L & 2000 & Nov 26, 2008 \\
\hline 12 & 11 & M & R & 1999 & Mar 6, 2008 \\
\hline 13 & 8 & M & L & 2001 & Sep 18, 2008 \\
\hline 14 & 8 & M & L & 2002 & Mar 14, 2007 \\
\hline
\end{tabular}

For the radiographic assessment, we used the most recent examinations available in the patients' medical records. Lateral-view radiographs in which body weight was borne on both feet were analyzed. The mean time elapsed between the surgery and the control radiograph used in this study was 6.53 years (range: two to twelve years). The morphological characteristics of 
the talus of the operated foot were compared with the radiographic parameters of the normal contralateral foot. The presence of deformity of the talar dome and head, the height and length of the talus, the presence and degree of subluxation of the navicular, the changes to the Gissane angle and the trabecular bone pattern were assessed.

To analyze the deformity of the talar dome, we evaluated the sphericity of the tibiotalar joint, using a ruler of concentric rings in accordance with the Mose method. The feet studied were subdivided into three groups: (A) normal, allowing good joint mobility; (B) slightly flattened; (C) greatly altered or flat (Figure 1).

In order to assess the deformity of the head, the feet were divided into three groups according to the sphericity of the talonavicular joint: (A) convex; (B) planar; (C) concave (Figure 2).

We did not find in the literature any preestablished parameter for evaluating the height or length of the talus. After testing several techniques for making such measurements, with assistance from a radiologist, we found that the standardization and reproducibility between different radiographs was easiest with the following techniques:

Height measurement (Figure 3):

1. Identify the apex of the lateral process of the talus (point 1);
2. Draw a straight line parallel to the ground, tangential to this point (straight line 2);

3. From point 1 , draw a straight line perpendicular to straight line 2 (straight line 3 ), as far as the intersection point with the joint surface of the talar dome (point 4));

4. The height of the talus is given by the length of straight line 2 from point 1 to point

Length measurement (Figure 4):

1. Identify the highest point (point 1) and the lowest point (point 2) of the joint surface of the talus, in the talonavicular joint, on the lateral-view radiograph;

2. Draw a straight line joining points 1 and 2 (straight line 3);

3. Identify the midpoint of straight line 3 (point 4);

4. Identify the most posterior point of the posterior process of the talus, not including the os trigonum (point 5);

5. Draw a straight line (straight line 6) from point 5 , going through point 4 and as far as the intersection with the joint surface of the talus, in the talonavicular joint (point 7);

6. The distance from point 5 to point 7 constitutes the length of the talus.

The height and length were measured in the affected foot and the contralateral foot. A simple percentage
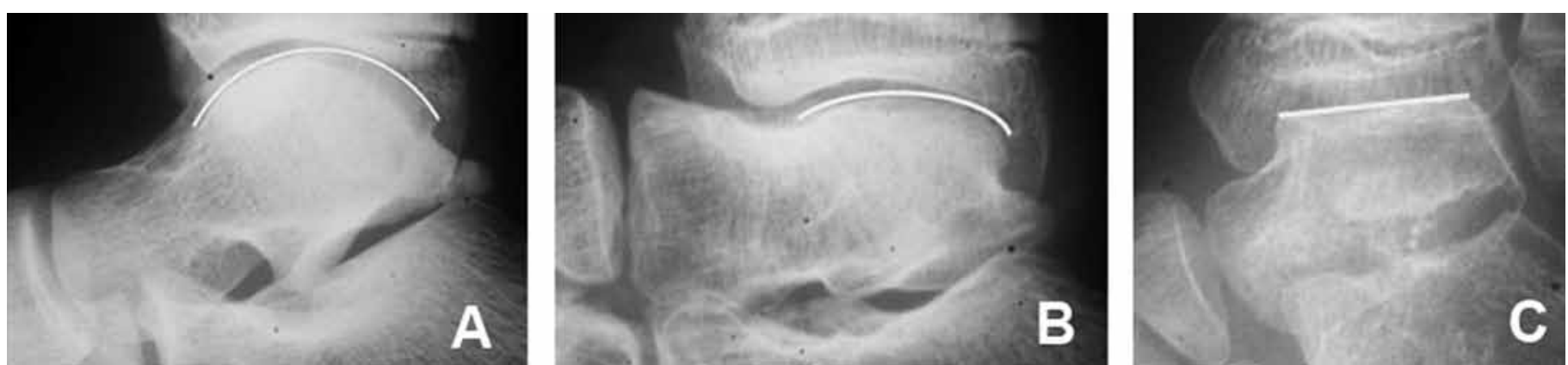

Figure 1 - Talar sphericity patterns: (A) normal, enabling good mobility of the joint; (B) slightly flattened; (C) greatly altered or flat.
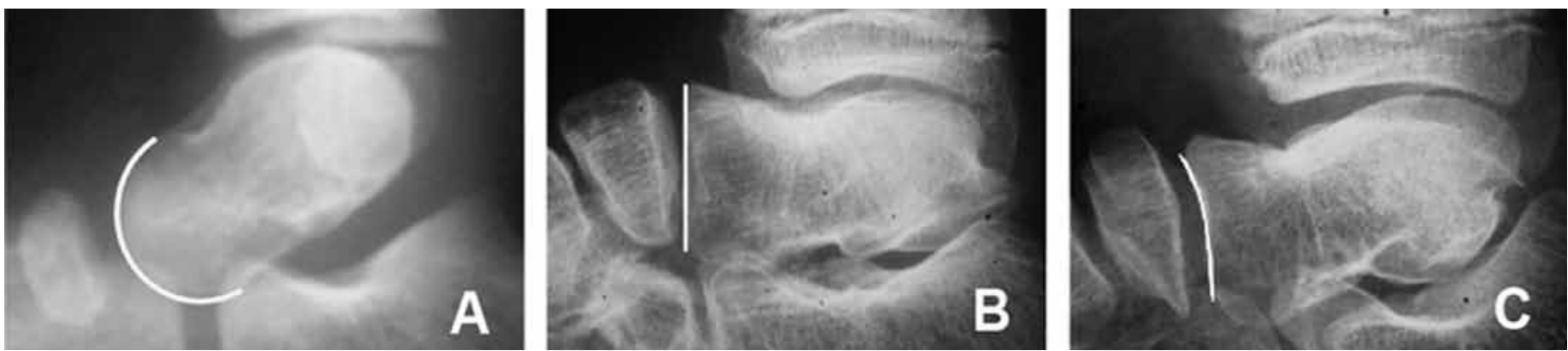

Figure 2 - Sphericity patterns for the talonavicular joint: talar head (A) convex; (B) planar; (C) concave. 


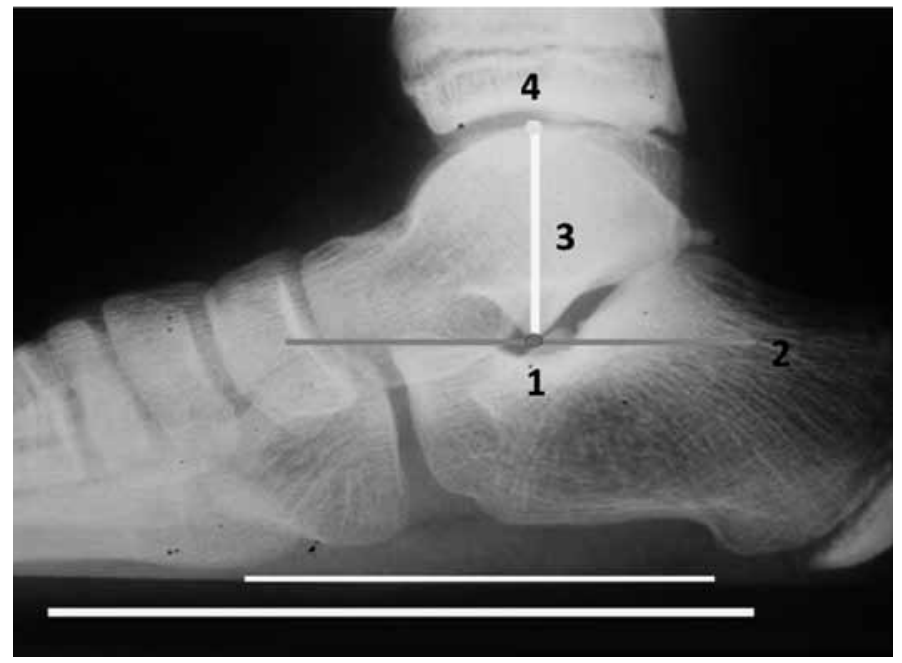

Figure 3 - Illustration of measurement of talar height (explained in text).

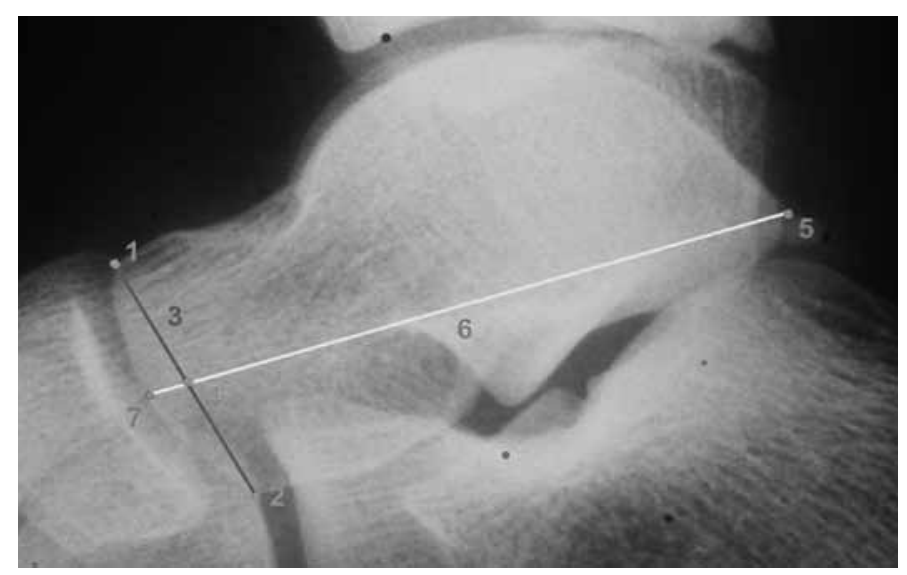

Figure 4 - Illustration of measurement of talar length (explained in text).

measurement was then obtained between these measurements.

Morphological abnormalities of the talus in the subtalar joint were evaluated by measuring the Gissane angle, which consists of the angle between the straight line tangential to the anterior joint facet and the straight line tangential to the posterior joint facet of the talus. This angle was measured in both feet of each patient and the difference between the affected foot and normal foot was then calculated.

To analyze the degree of subluxation of the navicular in the talonavicular joint, we established an index that was calculated as follows (Figure 5):

1. Identify the highest point (point 1) and the lowest point (point 2) of the joint surface of the talus in the talonavicular joint, on the lateral-view radiograph;

2. Draw straight lines joining points 1 and 2 (straight line 3);

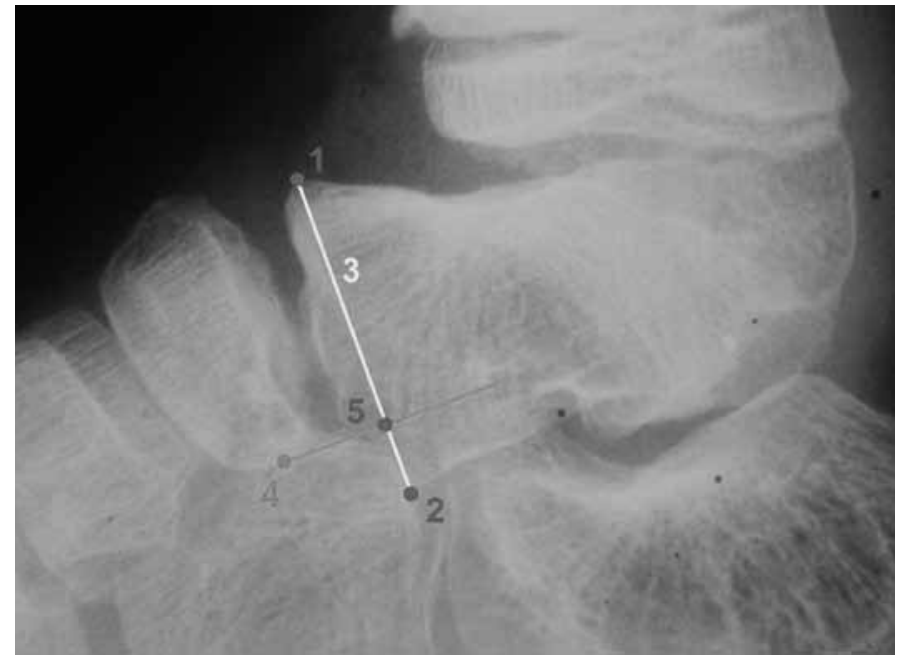

Figure 5 - Illustration of calculation of degree of navicular subluxation (explained in text).

3. Draw a straight line perpendicular to straight line 3 , tangential to the lowest point of the navicular (point 4);

4. The point where the two straight lines intersect is point 5;

5. The index is calculated by obtaining the ratio of the distances between points 2 and 5 , over the distance between points 1 and 2 .

To assess the trabecular bone pattern of the talus in the operated foot in relation to the normal foot, we divided the cases into three groups according to the predominant direction of the bone trabeculae in the radiographs studied: (A) horizontal: predominance of horizontal trabeculae, which is a pattern compatible with a normal talus; (B) mixed: presence of equally distributed vertical and horizontal trabeculae; (C) vertical: predominance of vertically orientated trabeculae.

\section{RESULTS}

The results from the radiographic evaluation are illustrated in Table 2.

Out of the 14 radiographs evaluated, $85.71 \%$ (12 patients) presented flattening of the talus head, in relation to the contralateral foot; $7.14 \%$ (one patient) presented convexity and $7.14 \%$ (one patient) presented concavity.

Regarding the deformity of the talar dome of the operated foot, $71.43 \%$ of the cases (10 patients) presented the elliptical form, $21.43 \%$ (three patients) had the planar form and $7.14 \%$ (one patient) had the spherical form. 
Table 2 - Data on patients regarding the results from the radiographic parameters evaluated.

\begin{tabular}{c|c|c|c|c|c|c|c}
\hline Patient & $\begin{array}{c}\text { Deformity of } \\
\text { head }\end{array}$ & $\begin{array}{c}\text { Deformity of } \\
\text { dome }\end{array}$ & $\begin{array}{c}\text { Height } \\
\text { ratio }\end{array}$ & Length ratio & $\begin{array}{c}\text { Trabecular } \\
\text { type }\end{array}$ & $\begin{array}{c}\text { Gissane } \\
\text { increase }\end{array}$ & $\begin{array}{c}\text { Navicular } \\
\text { subluxation rate }\end{array}$ \\
\hline 1 & Flattened & Planar & 0.86 & 0.79 & Mixed & $40^{\circ}$ & $25.12 \%$ \\
\hline 2 & Flattened & Elliptical & 0.92 & 0.84 & Mixed & $30^{\circ}$ & $41.17 \%$ \\
\hline 3 & Flattened & Elliptical & 0.57 & 0.84 & Vertical & $7^{\circ}$ & $8.02 \%$ \\
\hline 4 & Flattened & Planar & 0.95 & 0.77 & Vertical & $20^{\circ}$ & $22.29 \%$ \\
\hline 5 & Flattened & Spherical & 0.91 & 0.75 & Mixed & $20^{\circ}$ & $20.91 \%$ \\
\hline 6 & Convex & Elliptical & 0.98 & 0.87 & Mixed & $24 \%$ & $58.80 \%$ \\
\hline 7 & Concave & Elliptical & 0.86 & 0.86 & Mixed & $30^{\circ}$ & $12.33 \%$ \\
\hline 8 & Flattened & Elliptical & 0.82 & 0.88 & Vertical & $2 \%$ & $33.74 \%$ \\
\hline 9 & Flattened & Elliptical & 0.87 & 0.61 & Vertical & $30^{\circ}$ & $17.33 \%$ \\
\hline 11 & Flattened & Elliptical & 0.76 & 0.74 & Vertical & $22^{\circ}$ & $6.43 \%$ \\
\hline 12 & Flattened & Elliptical & 0.75 & 0.88 & Mixed & $20^{\circ}$ & $17.06 \%$ \\
\hline 13 & Flattened & Elliptical & 0.84 & 0.86 & Vertical & $8^{\circ}$ & $23.71 \%$ \\
\hline 14 & Flattened & Planar & 0.7 & 0.80 & Vertical & $26^{\circ}$ & $59.75 \%$ \\
\hline & Flattened & Elliptical & 0.65 & 0.61 & Vertical & $10^{\circ}$ & $22.19 \%$ \\
\hline
\end{tabular}

All the cases presented reductions in length of at least $10 \%$ and, of these, half presented reductions of at least $20 \%$. The ratio of talus length in the operated foot to talus length in the contralateral foot ranged from 0.61 to 0.88 , with a mean of $0.7929(\mathrm{SD}=0.0905)$.

The height of the talus in the operated feet was less than in the contralateral foot in all the cases evaluated. The ratio ranged from 0.57 to 0.98 , with a mean of $0.8171(\mathrm{SD}=0.11796)$, with a reduction of at least $10 \%$ in most cases $(71.43 \%)$.

In the operated feet, no talus presented a normal trabecular pattern, i.e. predominantly horizontal: $57.14 \%$ of them presented a mixed pattern (vertical and horizontal) and $42.86 \%$ presented a predominantly vertical pattern.

The Gissane angle was greater in all the operated feet, in comparison with the contralateral foot. This increase ranged from 2 to $40^{\circ}$, with a mean of $20.64^{\circ}$, and in 10 cases $(71.43 \%)$, the increase was at least $20 \%$.

All the cases presented some degree of subluxation of the navicular in the talonavicular joint, and its rate ranged from 6.43 to $59.75 \%$, with a mean of $26.34 \%$ $(\mathrm{SD}=16.66 \%)$. Out of the 14 feet assessed, nine $(64.28 \%)$ presented navicular elevation of at least $20 \%$.

\section{DISCUSSION}

Many complications relating to surgery with extensive release of soft tissues for treating clubfoot have been described in the literature. However, there are no well-defined radiographic criteria for diagno- sing necrosis and other alterations of the talus in this disease. Thus, we established our own radiographic parameters based on the atypical morphological characteristics encountered.

There have been reports on changes to the shape of the talus following treatment for clubfoot, both with surgical and with nonsurgical techniques ${ }^{(5-7)}$. In 1981 , Ponseti et $\mathrm{al}^{(8)}$ reported changes to the total length and shape of the talar dome and head, in their wellestablished protocol for treatment using serial plaster casts. Cummings et al ${ }^{(9)}$ reported "bone-within-thebone" alterations, with flattening of the dome and hypoplasia of the talar head and neck, after surgical treatment using McKay's technique. Several other authors have also reported a varying number of talar abnormalities with different treatment techniques.

We observed greater incidence $(100 \%)$ of radiographic abnormalities of the talus, compared with the usual incidence previously described $(27-74 \%)^{(7-10)}$. This was probably a consequence of the technique used by the surgeon at the time, which consisted of McKay's technique with extensive peritalar release and significant damage to the arterial supply to the talus.

Because of the scarcity of descriptive radiographic anatomical studies on talar deformity in cases of clubfoot, we put forward some parameters for a more precise evaluation of this complication. These parameters are the height and length of the talus, degree of talonavicular subluxation, Gissane angle, characteristics of the deformity of the talar head and 
dome and trabecular bone pattern. The Gissane angle is already a well-established measurement within orthopedics. The height and length of the talus and the degree of talonavicular subluxation were measured using a system of straight lines drawn from objective anatomical points, and were compared with the same measurements on the contralateral foot, by means of simple percentages. This method was devised in collaboration with a radiologist who specializes in studies on the musculoskeletal system.

The other parameters, i.e. the characteristics of the deformity of the talar head and dome and the trabecular bone pattern, are determined through direct observation, in comparison with the normal anatomy of the unaffected foot. In cases of deformity of the talar head and dome, the parameters can also be measured objectively, by means of an instrument as simple as the Mose ruler of concentric rings. We believe that these would be simple and objective parameters that would be easy to apply and would probably present a good reproducibility rate.

One possible limiting factor in our study is perhaps the small absolute number of patients included, as well as the lack of clinical correlation with the radiographic results, which we intend to address in a subsequent study.

Kalenderer et al ${ }^{(11)}$ observed significant changes in talar length and height in all the patients in their series, along with deformities of the dome and talonavicular joint in the great majority of the cases. However, there was no significant clinical-radiographic correlation with these changes, given that all their patients achieved good results, without limitations on their activities. These patients and their parents expressed their satisfaction with the functional and cosmetic results. Segev et $\mathrm{ll}^{(12)}$ also found significant alterations in talus size in feet that were treated conservatively, without comparable clinical impairment.

We consider that it is very important to quantify and establish descriptive parameters for talar deformity, because of its clinical implication. Flattening of the talar dome alters the tibiotarsal joint with consequent diminution of the flexion-extension of the foot. Talonavicular deformity and subluxation is associated with diminution of midfoot mobility. Over the long term, osteoarthrosis may become established, and this may evolve with pain and functional limitation.

However, we cannot affirm that these alterations result from avascular necrosis, since they may simply represent a further characteristic of this disease. One point favoring this theory is that some of these deformities occur in feet undergoing both conservative treatment and surgical treatment. Moreover, these alterations do not fit within the characteristics usually observed in cases of osteonecrosis of the talus, which show increased density, fragmentation and collapse ${ }^{(9)}$.

\section{CONCLUSION}

Talar abnormalities were observed in $100 \%$ of the feet treated using McKay's technique, among this sample. Establishment of radiographic parameters to describe and quantify talar deformities was shown to be viable, by means of simple and easily performed techniques.

\section{REFERENCES}

1. Dobbs MB, Gurnett CA. Update on clubfoot: etiology and treatment. Clin Orthop Relat Res. 2009;(467):1146-53.

2. Mahmoodian R, Leasure J, Gadikota H, Capaldi F. Mechanical properties of human fetal talus. Clin Orthop Relat Res. 2009;(467):1186-94.

3. Pearce DH, Mongiardi CN, Fornasier VL, Daniels TR. Avascular necrosis of the talus: a pictoril essay. Radiographics. 2005;25(2):399-410.

4. McKay DW. New concept of and approach to clubfoot treatment: section II correction of the clubfoot. J Pediatr Orthop. 1983;3(1):10-21.

5. McKay DW. New concept of and approach to clubfoot treatment: section I principles and morbid anatomy. J Pediatr Orthop. 1982;2(4):347-56.

6. Napiontek M. Clinical and radiographic appearance of congenital talipes equinovarus after successful nonoperative treatment. J Pediatr Orthop. 1996; 16(1):67-72.

7. Farsetti P, De Maio F, Russolillo L, Ippolito E. CT study on the effect of di-

Rev Bras Ortop. 2011;46(3):293-98

fferent treatment protocols for clubfoot pathology. Clin Orthop Relat Res. 2009;(467):1243-9.

8. Ponseti IV, El-Khoury GY, Ippolito E, Weinstein SL. A radiographic study of skeletal deformities in treated clubfeet. Clin Orthop Relat Res. 1981;(160):30-42.

9. Cummings RJ, Bashore CJ, Bookout CB, Elliott MJ. Avascular necrosis of the talu after McKay clubfoot release for idiopathic congenital clubfoot. J Pediatr Orthop. 2001;21(2):221-4.

10. Ippolito E, Fraracci L, Farsetti P, Di Mario M, Caterini R. The influence of treatment on the pathology of club foot. J Bone Joint Surg. 2004;86(4):574-80.

11. Kalenderer $O$, Reisoglu A, Turgut A, Agus $H$. Evaluation of clinical and radiographic outcomes of complete subtalar release in clubfoot treatment. J Am Podiatr Med Assoc. 2008;98(6):451-6.

12. Segev E, Yavor A, Ezra E, Hemo Y. Growth and development of tarsal and metatarsal bones in successfully treated congenital idiophatic clubfoot: early radiographic study. J Pediatr Orthop B. 2009;18(1):17-21. 